\title{
On the solution of the Kompaneets equation in cosmological context: a numerical code to predict the CMB spectrum under general conditions
}

\author{
C. Burigana, P. Procopio, and A. De Rosa \\ INAF - IASF Bologna, Via P. Gobetti 101, 40129 Bologna
}

\begin{abstract}
Interpretation of current and future data calls for a continuous improvement in the theoretical modeling of CMB spectrum. We describe the new version of a numerical code, KYPRIX, specifically written to solve the Kompaneets equation in a cosmological context under general assumptions. We report on the equation formalism, and structure and computational aspects of the code. New physical options have been introduced in the current code version: the cosmological constant in the terms controlling the general expansion of the Universe, the relevant chemical abundances, and the ionization history, from recombination to cosmological reionization. We present some of fundamental tests we carried out to verify the accuracy, reliability, and performance of the code. All the tests demonstrate the reliability and versatility of the new code version and its accuracy and applicability to the scientific analysis of current CMB spectrum data and of much more precise measurements that will be available in the future.
\end{abstract}

Keywords. Cosmology, cosmic microwave background, radiative processes, numerical methods

\section{Introduction}

Ground-based, balloon-born, and space experiments, and, in particular, the COBE/ FIRAS results (Fixsen et al., 1996), confirm that only very small deviations from a Planckian shape can be present in the CMB spectrum. Therefore, current and future CMB absolute temperature experiments aim at discovering very small distortions that are predicted to be associated with the cosmological reionization process or that could be generated by different kinds of earlier processes. Interpretation of future data (Fixsen \& Mather, 2002; Burigana \& Salvaterra, 2003, Mather, 2009) calls for a continuous improvement in the theoretical modeling of CMB spectrum. We describe the new version (Procopio \& Burigana, 2009) of a numerical code, KYPRIX, specifically written to solve the Kompaneets equation in a cosmological context and first implemented in the years 1989-1991, to accurately compute the CMB spectral distortions under general assumptions. After the presentation of the necessary formalism and of the main structure of the code, we describe here the new physical options introduced in the current version.

\section{The Kompaneets equation in cosmological context}

Physical processes occurring at redshifts $z<z_{\text {therm }}$ may leave imprints on the CMB spectrum. Thus, it carries crucial informations on physical processes occurring during early cosmic epochs (see e.g. Danese \& Burigana, 1993 and references therein) and the comparison between models of CMB spectral distortions and CMB absolute temperature measures can constrain their physical parameters (Burigana et al., 1991b). 
The time evolution of the photon occupation number, $\eta(\nu, t)$, under the effect of Compton scattering and of photon production processes, radiative Compton (RC) (Gould, 1984), bremsstrahlung (B) (Karzas \& Latter, 1961; Rybicki \& Lightman, 1979), plus other possible photon emission/absorption contributions $(\mathrm{EM}) \dagger$, is well described by the complete Kompaneets equation (Kompaneets, 1956; Burigana et al., 1995):

$$
\frac{\partial \eta}{\partial t}=\frac{1}{\phi} \frac{1}{t_{C}} \frac{1}{x^{2}} \frac{\partial}{\partial x}\left[x^{4}\left[\phi \frac{\partial \eta}{\partial x}+\eta(1+\eta)\right]\right]+\left[\frac{\partial \eta}{\partial t}\right]_{R C}+\left[\frac{\partial \eta}{\partial t}\right]_{B}+\left[\frac{\partial \eta}{\partial t}\right]_{E M},
$$

where $t_{C}=t_{\gamma e} m_{e} c^{2} /\left(k T_{e}\right) \simeq 4.5 \times 10^{28}\left(T_{0} / 2.7 K\right)^{-1} \phi^{-1} \widehat{\Omega}^{-1}(1+z)^{-4} \sec , t_{\gamma e}=1 /\left(n_{e} \sigma_{T} c\right)$ is the photon-electron collision time, $\phi=\left(T_{e} / T_{r}\right), T_{e}$ and $T_{r}=T_{0}(1+z)$ being respectively the electron and the CMB radiation temperature; and $x=h \nu_{0} / k T_{0}=h \nu_{0}(1+$ $z) / k T_{0}(1+z)$ is a dimensionless, redshift independent, frequency ( $\nu_{0}$ being the present frequency), being $\epsilon_{r 0}=a T_{0}^{4}$ the radiation energy density today. This equation is coupled to the time differential equation governing the electron temperature evolution for an arbitrary radiation spectrum in the presence of the above processes plus adiabatic cooling and possible external heating sources, $q=a^{-3}(d Q / d t)$,

$$
\frac{d T_{e}}{d t}=\frac{T_{e q, C}-T_{e}}{(27 / 28) t_{e \gamma}}+\left[\frac{d T_{e}}{d t}\right]_{R C, B, E M}-\frac{2 T_{e}}{t_{e x p}}+\frac{(32 / 27) q}{3 n_{e} k}
$$

here $T_{e q, C}=\left[h \int \eta(1+\eta) \nu^{4} d \nu\right] /\left[4 k \int \eta \nu^{3} d \nu\right]$ is the Compton equilibrium electron temperature (Peyraud, 1968, Zeldovich \& Levich, 1970), $t_{e \gamma}=3 m_{e} c /\left(4 \sigma_{T} \epsilon_{r}\right), \epsilon_{r} \simeq \epsilon_{r 0}(1+z)^{4}$.

\section{The numerical code KYPRIX}

The numerical code KYPRIX was written to overcome the limited applicability of analytical solutions and to get a precise computation of the evolution of the photon distribution function for a wide range of cosmic epochs and for many cases of cosmological interest (Burigana et al., 1991a). KYPRIX makes use of the NAG libraries (NAG Ltd, 2009) and of a lot of numerical algorithms, available to the scientific community or written specifically for code dedicated tasks. The D03PCF routine of the current version of the NAG release has been used to reduce the Kompaneets equation into a system of ordinary differential equations (Dew \& Walsh, 1981; Berzins et al., 1989; Skeel \& Berzins, 1990). The numerical framework is the same of the D03PGF routine, no longer available, used in the first versions of KYPRIX, but they come from different technical implementations and present remarkable differences.

The code is divided in several sections. 1) Main program, for the defiinition of: choice of the physical processes, choice of the cosmological parameters, initial conditions, characteristics of the numerical integration (accuracy, number of points of the grid), time interval of interest, choice of the boundary conditions, chemical abundances, ionization history. 2) Subroutine PDEDEF, the subprogram where the problem is numerically defined. This subroutine is also divided in subsections to allow modifications in a simple and practical way. 3) Subroutine BNDARY, where the boundary conditions are numerically specified. 4) Subroutines and auxiliary functions to perform specific calculations.

During the numerical integration, some subprograms use the distribution function

$\dagger$ A process that in principle should be included is the cyclotron emission. On the other hand, for realistic values of cosmic magnetic field and CMB realistic distorted spectra, the cyclotron process never plays an important role for (global) CMB spectral distortions when ordinary and stimulated emission and absorption are properly taken into account (Zizzo \& Burigana, 2005). 
calculated at that time to compute $\phi$. The integrals to be computed are those that we find in the expression for $\phi_{e q, C}=T_{e q, C} / T_{r}$. In this calculation, the integration range is obviously the interval considered for the problem: $A \leqslant X \leqslant B$ (that, in terms of mesh ordering, corresponds to the range between 1 and NPTS or NPTS - 1; the adopted grid uses $X=\log x$ ). For computing these integrals, all the points of the grid are used. The integration is based on the NAG D01GAF routine, suitable for tabulated functions. Thanks to the opportunity of having the correct value of $\phi$ for each time step, the update of the boundary conditions can be physically motivated. We implemented in the code the possibility to adopt a particular case of Neumann boundary conditions: the requirement that the current density, in the frequency space, is null at the boundaries of the integration range (Chang \& Cooper, 1970): $[\phi \partial \eta / \partial x+\eta(1+\eta)]_{x=x_{\text {min }}, x_{\text {max }}}=0$.

\section{Physical options of the code}

The code KYPRIX has been written to solve the Kompaneets equation in many kinds of situations. The physical processes that can be considered are: Compton scattering, bremsstrahlung, radiative Compton scattering, sources of photons, energy injections without photon production, energy exchanges (heating or cooling processes) associated to $\phi \neq 1$ at low redshifts, radiative decays of massive particles, and so on (see e.g. Danese \& Burigana, 1993 for some applications). This code could be easily implemented to consider other kinds of physical processes. Various kinds of initial conditions for the problem can be considered and many of them have been already implemented in KYPRIX. The first obvious case is a pure Planckian spectrum. Several ways to model an instantaneuos heating implying deviations from the Planckian spectrum have been introduced: a pure Bose-Einstein (BE) spectrum or a BE spectrum modified to become Planckian at low frequencies; a grey-body spectrum; a superposition of blackbodies.

We have then updated the numerical integration code KYPRIX to include the cosmological constant and the curvature in the terms controlling the general expansion of the Universe, thus making the code suitable to be accurately applied at late ages. Remarkable examples are spectral distortions associated to the reionization of the Universe (Burigana et al., 2008) that, in typical astrophysical scenarios, starts $z<10-20$.

In this new version of the code it is possible to choose the primordial abundances of hydrogen, $H$, and helium, $H e$, and to consistently compute $n_{e}$. Denoting with $f_{H}$ the fraction in mass of primordial $H$, since $n_{e}=n_{H}+2 n_{H e}$, we have $n_{e}^{\text {free }}=n_{e}^{\text {tot }}=$ $\left[\left(1+f_{H}\right) / 2\right]\left(\rho_{b} / m_{b}\right)$. This obviously impacts the physical processes involved in the code. Note that Compton scattering, radiative Compton, and bremsstrahlung depend linearly on $n_{e}^{f r e e}$ (for bremsstrahlung, the dependence on the densities of nuclei is now explicit).

We have then included the accurate modeling of the ionization history in the current implementation of KYPRIX. In particular, the fraction of each state of ionization of the relevant elements $(H$ and $H e$ ) has been implemented. Given the electron ionization fraction, $\chi_{e}$, from the charge conservation law we have a constraint on the number of the free ions in the considered plasma. The simplest way to take count of them in the code is to assume an equal fraction of ionization for $H$ and $H e$. A more accurate treatment of the physics of reionization/recombination processes implemented in the code is based on the Saha equation. The code can also ingest a table with the desired evolution of $\chi_{e}$ and, as necessary for a physical modeling of cosmological reionization, of the electron temperature. Obviously, we have implemented the best way to perform the exact calculation of the rates of considered processes in scenarios involving reionization/recombination which is that of using a co-running code, coupled to KYPRIX, able to supply the ionization fraction for all the species. For the recombination process, we developed an interface that 
allows to call an external program, in our case RECFAST (Seager et al., 1999), and run it with the same cosmological parameters selected for KYPRIX.

\section{Code porting, tests, and conclusion}

We successfully finalized the porting of the code KYPRIX through different platforms and environments, i.e. VAX machines (original platform, disused), DEC machines, IBM Power5/Power6 and Intel based machines. All the working machines in which the code was run on have a 64 bit OS. We carried out fundamental tests to verify the accuracy, reliability, and performance of the code: energy conservation, time behavior of electron temperature, comparison between the results obtained with both the update and the original version of the code, and properties of the free-free distortions relevant at long wavelengths. All the tests demonstrate the reliability and versatility of the new code version and its accuracy and applicability to the scientific analysis of current CMB spectrum data and of much more precise measurements that will be available in the future.

\section{Acknowledgements}

Calculations have been carried out on the IBM SP5/512 machine at CINECA-Bologna and on the computational facilities at INAF-IASF Bologna. We warmly thank M. Genghini and C. Gheller for the technical support related to the machines used. We acknowledge the use of the code RECFAST. The ASI contract I/016/07/0 "COFIS" is acknowledged.

\section{References}

Berzins, M. 1990, in Scientific Software Systems, eds. J. C. Mason \& M. G. Cox, 59, Chapman \& Hall, p. 59

Berzins, M., Dew, P. M., \& Furzeland, R. M. 1989, Appl. Numer. Math., 5, 375

Burigana, C., Danese, L., \& de Zotti, G. 1991, A\&A, 246, 49

Burigana, C., Danese, L., \& de Zotti, G. 1991, ApJ, 379, 1

Burigana, C., de Zotti, G., \& Danese, L. 1995, A\&A, 303, 323

Burigana, C. \& Salvaterra, R. 2003, MNRAS, 342, 543

Burigana, C., et al. 2008, MNRAS, 385, 404

Chang, J. S. \& Cooper, G. 1970, J. Comput. Phys., 6, 1

Danese, L., Burigana, C. 1993, in Present and Future of the Cosmic Microwave Background, Lecture in Physics, Vol. 429, eds. J. L. Sanz et al., Springer, Heidelberg (FRG), p. 28

Dew, P. M., Walsh, J. 1981, ACM Trans. Math. Software, 7, 295

Fixsen, D. J., et al. 1996, ApJ, 473, 576

Fixsen, D. J., Mather, J. C. 2002, ApJ, 581, 817

Gould, R. J. 1984, ApJ, 285, 275

Karzas, W. J., Latter, R. 1961, ApJS, 6, 167

Kompaneets, A. S. 1956, Zh. Eksp. Teor. Fiz., 31, 876 [Sov. Phys. JEPT, 4, 730, (1957)]

Mather, J. C. 2009, in Questions of Modern Cosmology - Galileo's Legacy, eds. M. D'Onofrio \& C. Burigana, Springer, Sect. 5.3.1, p. 435

The Numerical Algorithms Group Ltd 2009, Oxford, UK

Peyraud, N. 1968, Physics Letters A, 27, 410

Procopio, P. \& Burigana, C. 2009, A\&A, 507, 1243

Rybicki G. B. \& Lightman A. P. 1979, Radiative processes in astrophysics, Wiley, New York

Seager, S., Sasselov, D. D., Scott, D. 1999, ApJ, 523, L1

Skeel, R. D. \& Berzins, M. 1990, SIAM J. Sci. Statist. Comput., 11(1), 1

Zeldovich, Y. B. \& Levich, E. V. 1970, Zh. Eksp. Teor. Fiz., 11, 57

Zizzo, A. \& Burigana, C. 2005, New Astronomy, 11, 1 